\title{
How Solvent Affects $\mathrm{C}-\mathrm{H}$ Activation and Hydrogen Production Pathways in Homogeneous Ru-Catalyzed Methanol Dehydrogenation Reactions
}

\author{
Vivek Sinha, ${ }^{\dagger, \S}$ Nitish Govindarajan, ${ }^{*}, \S_{\odot}$ Bas de Bruin, ${ }^{* \dagger} \dagger$ and Evert Jan Meijer ${ }^{\ddagger}$ \\ ${ }^{\dagger}$ Homogenous, Supramolecular and Bio-Inspired Catalysis, Van 't Hoff Institute for Molecular Sciences, University of Amsterdam, \\ Science Park 904, 1098 XH, Amsterdam, The Netherlands \\ ${ }^{\ddagger}$ Amsterdam Center for Multiscale Modeling and Van 't Hoff Institute for Molecular Sciences, University of Amsterdam, Science \\ Park 904, 1098 XH, Amsterdam, The Netherlands
}

\section{Supporting Information}

ABSTRACT: Insights into the mechanism of the catalytic cycle for methanol dehydrogenation catalyzed by a highly active PNP pincer ruthenium complex in methanol solvent are presented, using DFT-based molecular dynamics with an explicit description of the solvent, as well as static DFT calculations using microsolvation models. In contrast to previous results, we find the amido moiety of the catalyst to be permanently protonated under catalytic conditions. Solvent molecules actively participate in crucial reaction steps and significantly affect the reaction barriers when compared to pure gas-phase models, which is a direct result of the enhanced solvent stabilization of methoxide anion intermediates. Further, the calculations reveal that this system does not operate via the commonly assumed Noyori-type outer-sphere metal-ligand cooperative pathway. Our results show the importance of incorporating a molecular description of the solvent to gain a deeper and accurate understanding of the reaction pathways, and stress on the need to involve explicit solvent molecules to model complex catalytic processes in a realistic manner.

KEYWORDS: explicit solvent, methanol dehydrogenation, $\mathrm{C}-\mathrm{H}$ activation, $\mathrm{H}_{2}$ formation, molecular dynamics, density functional theory
$\mathrm{D}$ evelopment of a hydrogen economy is a promising path to address increasing global energy needs. The primary energy carrier in such an economy, dihydrogen, has a high gravimetric energy density and provides clean and carbon-free conversion. However, the use of dihydrogen as a fuel is limited by its low volumetric energy density under ambient conditions. Moreover, molecular hydrogen is difficult to produce, store and transport. Liquid organic fuels (LOFs), such as alcohols, provide a viable alternative in this regard. LOFs work on the principle of a reversible hydrogen storage mechanism. For example, three equiv of $\mathrm{H}_{2}$ can be stored in one molecule of $\mathrm{CO}_{2}$ by the reduction of $\mathrm{CO}_{2}$ to $\mathrm{MeOH}$ (and water). Systems that can perform reversible hydrogenation/dehydrogenation of $\mathrm{CO}_{2} / \mathrm{MeOH}$ can play a central role in establishing a hydrogen economy with $\mathrm{MeOH}$ as the primary energy carrier. Homogeneous molecular catalysts offer high selectivity and activity under ambient conditions to realize these conversions in an efficient manner. Various systems have been developed for conversion of $\mathrm{MeOH}$ to $\mathrm{CO}_{2}$ and $\mathrm{H}_{2},{ }^{1-7}$ and the reverse reaction as well. $^{8-11}$

Beller and co-workers reported the first catalytic system for low temperature aqueous methanol dehydrogenation using a ruthenium pincer complex $\mathbf{1}^{\prime}$ ( $\mathrm{RuH}(\mathrm{CO}) \mathrm{Cl}(\mathrm{HN}$ $\left.\left.\left(\mathrm{C}_{2} \mathrm{H}_{4} \mathrm{P}^{i} \mathrm{Pr}_{2}\right)_{2}\right)\right)$ as the catalytic precursor. ${ }^{1}$ The active catalyst complex $\mathbf{1}$ is capable of dehydrogenation of methanol-water mixtures with a turnover frequency (TOF) of $4700 \mathrm{~h}^{-1}$ and is highly stable (TON > 350000$)$. A number of other homogeneous (pincer and nonpincer type) $\mathrm{Fe}, \mathrm{Ru}, \mathrm{Ir}$, and Mn catalysts bearing $\mathrm{R}_{2} \mathrm{~N}$ amido donors (sometimes proposed to be in situ generated from $\mathrm{R}_{2} \mathrm{NH}$ amine donors) in a chelating ligand framework have also been reported, ${ }^{2,5,12-14}$ demonstrating the versatility of this class of complexes for methanol dehydrogenation.

For most of these catalysts outer-sphere Noyori-type mechanisms are typically proposed, involving proton transfer to the amido ligand and hydride transfer to the metal in the $\mathrm{MeOH}$ activation steps (Scheme 1). Similarly, a cooperative action of the thus formed amine ligand donor and the metal hydride resulting in hydride-proton coupling is generally assumed to account for $\mathrm{H}_{2}$ liberation and regeneration of the amido catalyst (Scheme 1). A shortcoming of most of these catalytic systems is the need for additives (base or Lewis acid cocatalyst) to achieve optimal performance. From a fundamental chemical perspective, a catalyst with a cooperative

Received: March 24, 2018

Revised: May 10, 2018

Published: June 12, 2018 
Scheme 1. Generally Proposed Noyori Type Cooperative Pathways for Acceptorless Dehydrogenation of Alcohols, in a Gas-Phase Context (left) and the Structure of Complex 1 Used in This Study (Right)

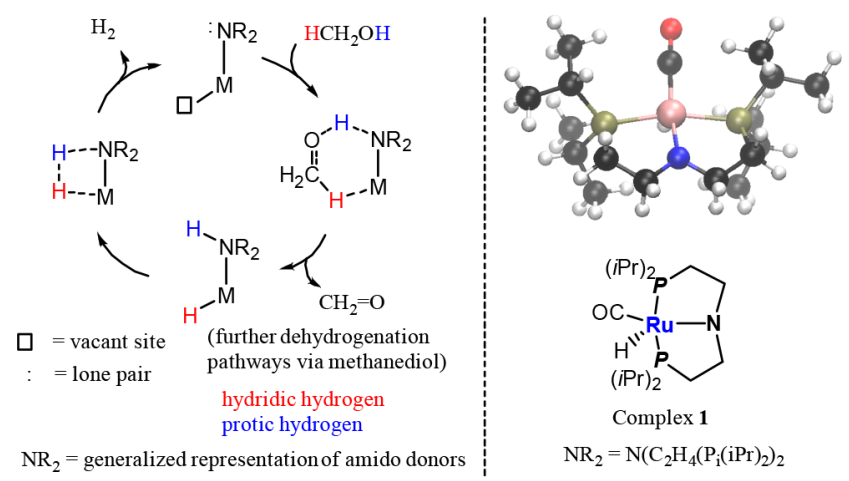

ligand, containing an internal amido-base and operating via an outer-sphere Noyori type mechanism ${ }^{15}$ for acceptorless dehydrogenation of an alcohol should not require any additives. As such, it is quite remarkable that Beller's RuPNP complexes were reported to operate via metal-ligand cooperative pathways, but yet perform optimally under highly basic conditions $(8 \mathrm{M} \mathrm{KOH})$. On the other hand, it has been demonstrated that $\mathrm{MeOH}$ dehydrogenation can be carried out under additive free conditions by choosing a different catalyst design principle, albeit with lower activity. ${ }^{2}$

Previous experimental and computational studies have rationalized the mechanism of dehydrogenation of methanol by complex 1 mostly in the framework of a Noyori-type mechanism. $^{16,17}$ This assumes a cooperative action of the amido ligand and the metal center in the proton and hydride transfer steps, with $\mathrm{MeOH}$ dehydrogenation proceeding via simultaneous hydride transfer to the metal upon deprotonation of the alcohol by the amido moiety. In the second step, $\mathrm{H}_{2}$ liberation from the catalyst is proposed to proceed via a proton-hydride coupling process with proton transfer from the amine donor to the metal hydride moiety, thus leading to regeneration of the amido moiety (Scheme 1 ). Note that, in all proposed mechanisms, protonation and deprotonation of the amido ligand are considered to be key steps of the catalytic cycle. Beller and co-workers proposed a base $\left(\mathrm{OH}^{-}\right)$assisted pathway for dehydrogenation of methanol by complex $1 .{ }^{16}$ The base was assumed to deprotonate the amine moiety of complex 2 to generate the anionic complex $2^{-}$. Subsequent rearrangement of the methoxide moiety in complex $2^{-}$was proposed to be followed by a hydride transfer to the metal center to produce $3^{-}$. Successive $\mathrm{N}$-protonation of the latter by a solvent molecule was anticipated to generate the hydrogenated complex 3 and formaldehyde, followed by dehydrogenation of complex 3 leading to evolution of $\mathrm{H}_{2}$ and regeneration of complex 1 to complete the catalytic cycle.

Given the fact that the experimental catalytic reactions are performed in protic solvent mixtures $\left(\mathrm{MeOH} / \mathrm{H}_{2} \mathrm{O}\right)$, thus providing a strong hydrogen bonding environment, one might anticipate that the previous mechanistic pictures based on gas phase DFT calculations are incomplete. The solvation effects of protic solvents are likely to have a strong influence on the proton and hydride transfer steps. Both the $\mathrm{MeOH}$ activation and the $\mathrm{H}_{2}$ formation steps might proceed via methoxide intermediates, where the strong hydrogen bonds with the negatively charged oxygen are crucial for a proper description of the relative stability of these intermediates. Moreover, in a protic solvent the solvent molecules might compete with the catalyst as the proton source. These scenarios could not be accounted for in previous computational studies, ${ }^{16,17}$ that did not account for solvent or incorporated solvent by a continuum model at most complemented by a single solvent molecule. The importance of an explicit (finite-temperature) description of the solvent has been demonstrated for other catalytic processes, ${ }^{18}$ including water splitting ${ }^{19-21}$ and transfer hydrogenation of ketones. ${ }^{22-24}$

To capture the effects of explicit solvent molecules, we employ a combined approach of ab initio molecular dynamics (DFT-MD) of an explicitly solvated periodic system, and static density functional theory (DFT) simulations of gas-phase and microsolvated systems. Full details are provided in the Supporting Information (SI). Our results reveal the active participation of solvent in this important reaction, as it has a nontrivial effect in the $\mathrm{C}-\mathrm{H}$ activation and hydrogen production pathways. On this basis, we propose a new catalytic pathway for methanol oxidation catalyzed by complex 1 (Scheme 2), significantly different from all previously proposed mechanisms.

An important first observation from our DFT-MD simulations with explicit solvent, either in water or in methanol, was the spontaneous protonation of the ligand nitrogen atom of complex $\mathbf{1}$ by a solvent molecule. The amido moiety remained protonated during the entire DFT-MD simulation $(25 \mathrm{ps})$. This process was accompanied by the coordination of an additional ligand such as $\mathrm{H}_{2} \mathrm{O}, \mathrm{CH}_{3} \mathrm{O}^{-}$or $\mathrm{MeOH}$ to the metal center, forming complexes $\mathbf{2}-\mathbf{H}_{2} \mathbf{O}^{+}, \mathbf{2}_{2 m}$, and $2-\mathrm{CH}_{3} \mathbf{O H}^{+}$respectively. This suggests that the solvent/ substrate saturated 18 valence electron octahedral ruthenium(II) species with a protonated amido moiety is a very stable species. To quantify the stability of this compound with respect to amido deprotonation, we determined the free energy change upon deprotonation of the $\mathrm{NH}$ moiety of complex $2-\mathrm{H}_{2} \mathrm{O}^{+}$by a solvent water molecule in aqueous solution using DFT-MD simulations, with the chosen reaction coordinate $(Q)$ imposing a proton transfer, involving cleavage of the $\mathrm{N}-\mathrm{H}$ bond and simultaneous formation of an $\mathrm{O}-\mathrm{H}$ bond (resulting in $\mathrm{H}_{3} \mathrm{O}^{+}$) (see SI). The computed value of $+35 \mathrm{kcal} \mathrm{mol}^{-1}$ (corresponding to a $\mathrm{p} K_{\mathrm{a}}$ of $\sim 25$ ), demonstrates the high stability of the N$\mathrm{H}$ bond, and the high basicity of the amido moiety in the PNP ligand (see SI for details). Therefore, even under very basic conditions complex $\mathbf{2}_{2 m}$ should remain protonated. In addition, using static DFT calculations the deprotonation of complex 2 by a microsolvated hydroxide moiety (that is present in such highly basic conditions) was found to be uphill by $+12 \mathrm{kcal}$ $\mathrm{mol}^{-1}$ (se SI), further demonstrating the high proton affinity of the amido moiety. Based on these results, it is evident that complex $\mathbf{2}_{2 m}$, with the protonated ligand nitrogen, is the resting state of this catalytic system under the experimentally applied reaction conditions ( $8 \mathrm{M} \mathrm{KOH}$ in a $9: 1 \mathrm{MeOH} / \mathrm{H}_{2} \mathrm{O}$ mixture).

Having established the resting state of this catalytic system, we first studied the oxidation of methanol catalyzed by complex $\mathbf{2}_{2 m}$ to form formaldehyde. Complex $\mathbf{2}_{2 m}$ undergoes an endergonic rearrangement to form complex $2_{2 m}^{\prime}$, where the methoxide moiety interacts with the metal center via an agostic $\mathrm{C}-\mathrm{H}-\mathrm{Ru}$ interaction (the methoxide is also hydrogen bonded to the $\mathrm{N}-\mathrm{H}$ moiety of the PNP ligand). The simulations indicate a direct hydride transfer from this methoxide moiety to the ruthenium center, resulting in the formation of $\mathrm{CH}_{2} \mathrm{O}$ and complex 3. Using DFT-MD with an explicit description of 
Scheme 2. Proposed Mechanism for Methanol Oxidation with Explicit Solvent (Top) and (Bottom) Important Complexes in This Study ${ }^{a}$
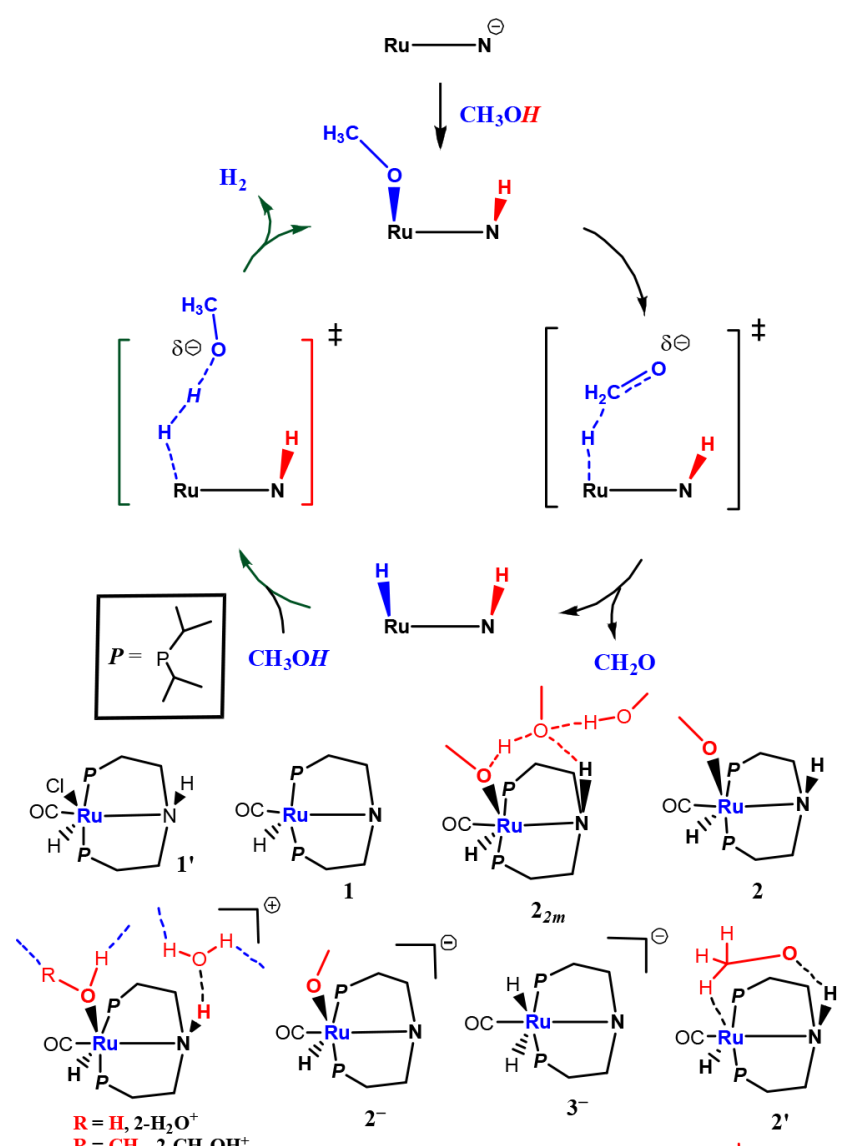

$$
\mathrm{R}=\mathrm{CH}_{3}, 2-\mathrm{CH}_{3} \mathrm{OH}^{+}
$$
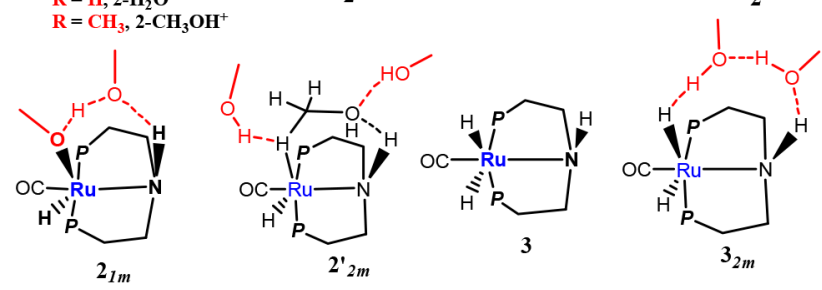

${ }^{a}$ Blue dashed lines in complex $\mathbf{2}-\mathrm{H}_{2} \mathrm{O}^{+} / \mathbf{2}-\mathrm{CH}_{3} \mathrm{OH}^{+}$show interactions with the solvent. The proton in the $\mathrm{NH}$ moiety of complexes $\mathbf{2}-\mathrm{H}_{2} \mathbf{O}^{+}$ and $2-\mathrm{CH}_{3} \mathrm{OH}^{+}$originates from the solvent. In the nomenclature of complexes in this manuscript, the subscript $1 m$ denotes interaction with one methanol solvent molecule, and the subscript $2 m$ denotes interactions with two methanol solvent molecules.

methanol solvent (see SI), we determined the free energy profile for this hydride transfer process using a biasing scheme, as shown in Figure 1 (top panel). The chosen hydride transfer reaction coordinate $(Q)$ that involves the cleavage of a $\mathrm{C}-\mathrm{H}$ bond and the simultaneous formation of a $\mathrm{Ru}-\mathrm{H}$ bond is shown in the inset of Figure 1 (top panel), and was varied between -0.9 and $1.0 \AA$. To quantify the role of the solvent, we also performed static DFT calculations of the corresponding hydride transfer pathway without (gas-phase), and with two explicit $\mathrm{MeOH}$ solvent molecules. Results for the minimum energy reaction pathway (MERP) are shown in Figure 1 (bottom panel). Note that the static DFT calculations are performed using a GGA functional (BP86) for a direct comparison with the DFT-MD results (BLYP). However, a hybrid functional like M06 was also explored and gave
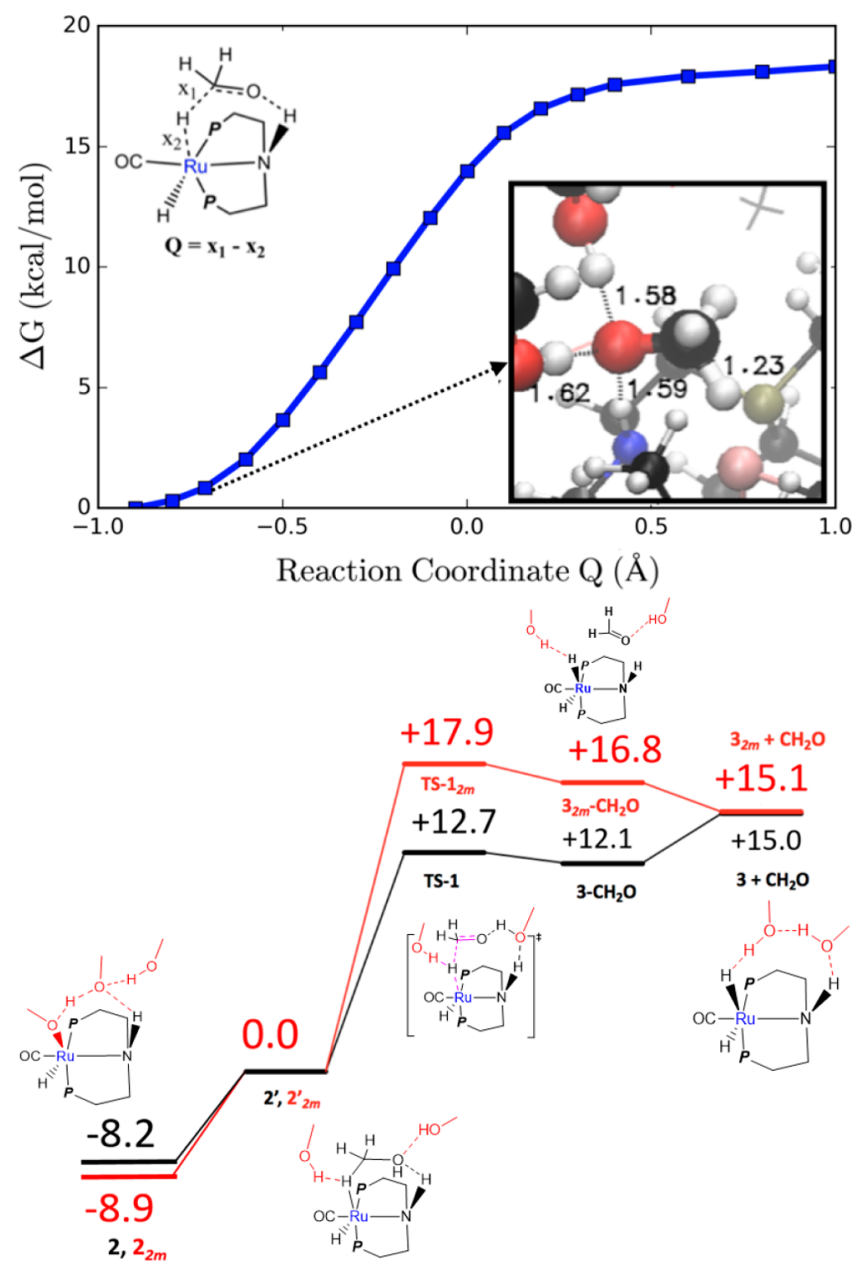

Figure 1. Free energy profile for methanol oxidation by hydride transfer from a methoxide anion to ruthenium, obtained from DFTMD simulation. The reaction coordinate $Q$ is specified in the left inset. The right inset shows a representative configuration at the initial stage $(Q=-0.9 \AA)$, showing the methoxide being stabilized by three strong hydrogen bonds (top) and (bottom). The calculated MERP (Gibbs free energy in $\mathrm{kcal} \mathrm{mol}^{-1}$ ) with static DFT (BP86/def2TZVP), using a gas-phase model (black) and an explicit microsolvation model with two additional $\mathrm{MeOH}$ molecules (red).

quantitatively similar results, as described in the SI. For a direct comparison with the free energy profile obtained via DFT-MD calculations, where the reference state is a solvated form of complex $2^{\prime}$, the TS barriers obtained using static DFT calculations in Figure 1 have been referenced with respect to complex $\mathbf{2}^{\prime}$ (and complex $\mathbf{2}_{2 m}^{\prime}$ for the microsolvated pathway).

The calculations show that the presence of explicit solvent molecules leads to a substantially higher transition state barrier (an increase $>5 \mathrm{kcal} \mathrm{mol}^{-1}$ ) for formaldehyde formation when compared to the gas-phase results. This effect can be directly attributed to the increased stabilization of the methoxide adduct in solution, due to its ability to form three strong hydrogen bonds (inset right panel in Figure 1). The reaction involves transfer of the negative charge from the oxygen on the methoxide moiety to the $\sigma^{*}$ orbital of the $\mathrm{C}-\mathrm{H}$ bond, leading to hydride transfer to the metal. Effective solvation of the methoxide anion by the $\mathrm{H}$-bond donating protic solvent molecules stabilizes the negative charge and thereby increases the barrier of the hydride transfer process (Figure 2). 

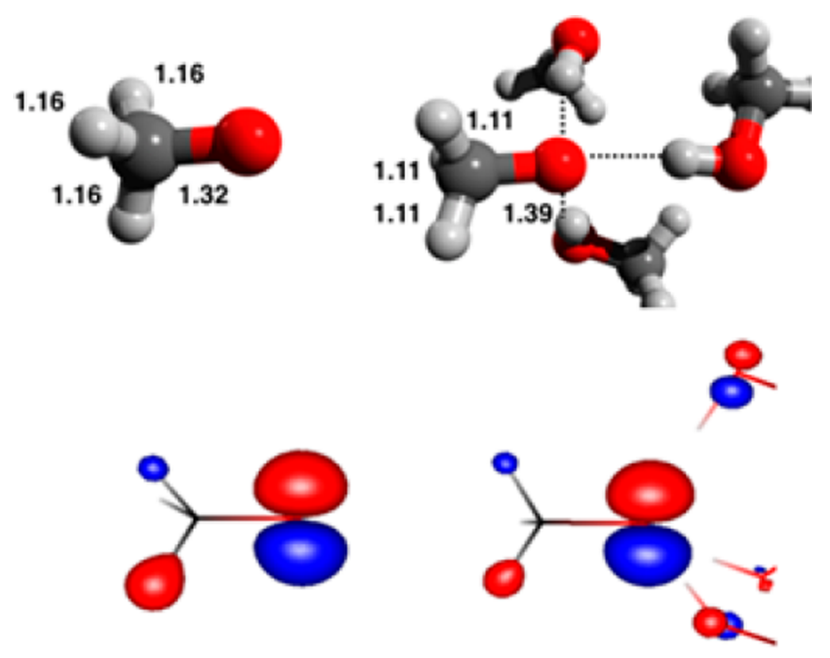

Figure 2. Comparison of the geometries and HOMOs of a methoxide anion and a methoxide anion hydrogen bonded to three $\mathrm{MeOH}$ molecules (distances in $\AA$ ).

Also for the hydrogen releasing steps under (micro)solvation conditions, we arrive at a different mechanism than previously proposed. Our calculations show that the reaction proceeds by a direct protonation of the metal hydride by a solvent molecule. The reaction barrier that we obtained for hydrogen production from complex 3 with explicit methanol solvent was $+7 \mathrm{kcal} \mathrm{mol}^{-1}$ (Figure 3, top). The chosen proton-transfer reaction coordinate $(\mathrm{Q})$ that involves the cleavage of the $\mathrm{O}-\mathrm{H}$ bond and the simultaneous protonation of the $\mathrm{Ru}-\mathrm{H}$ bond is shown in the inset of Figure 3 (top), and sampled between values of -0.4 and 1.6 $\AA$. Interestingly, the ligand nitrogen remains protonated throughout the reaction in solution, further demonstrating its strong proton affinity. To incorporate the effect of explicit solvent molecules in static DFT calculations, we studied the dehydrogenation process in the presence of two methanol molecules. One of the methanol molecules protonates the metal-hydride bond to form the dihydrogen complex $2_{2 m}-\mathbf{H}_{2}$. The resulting transition state barrier for dehydrogenation is significantly lower (a decrease of $6 \mathrm{kcal} \mathrm{mol}^{-1}$ ) than the corresponding activation barrier for dehydrogenation when hydrogen production is mediated by one methanol molecule (Figure 3, bottom). ${ }^{16,17}$ This remarkable effect can be attributed to the stabilization of the methoxide that is formed as the reaction proceeds, by hydrogen bonding to the protic solvent molecules. Release of dihydrogen and rearrangement of the methoxide species to coordinate with the metal center, regenerates the starting complex $2_{2 m}$ upon solvation by an extra methanol solvent molecule.

It is important to note at this point that the computed $\mathrm{MeOH}$ oxidation and hydrogen releasing steps under (micro)solvation conditions (Scheme 3) are substantially different from the previously reported Noyori-type cooperative dehydrogenation steps shown in Scheme 1. Clearly, the $\mathrm{NH}$ moiety does not get deprotonated under the applied conditions to function as a cooperative ligand, and it is neither directly involved in the dihydrogen releasing steps. However, the NH moiety of the PNP pincer ligand might still be important, as it could function as a supramolecular directing group, assisting the hydride transfer process to the metal by positioning the methoxide in a proper orientation by $\mathrm{H}$ bonding to the methoxide in the transition state for hydride
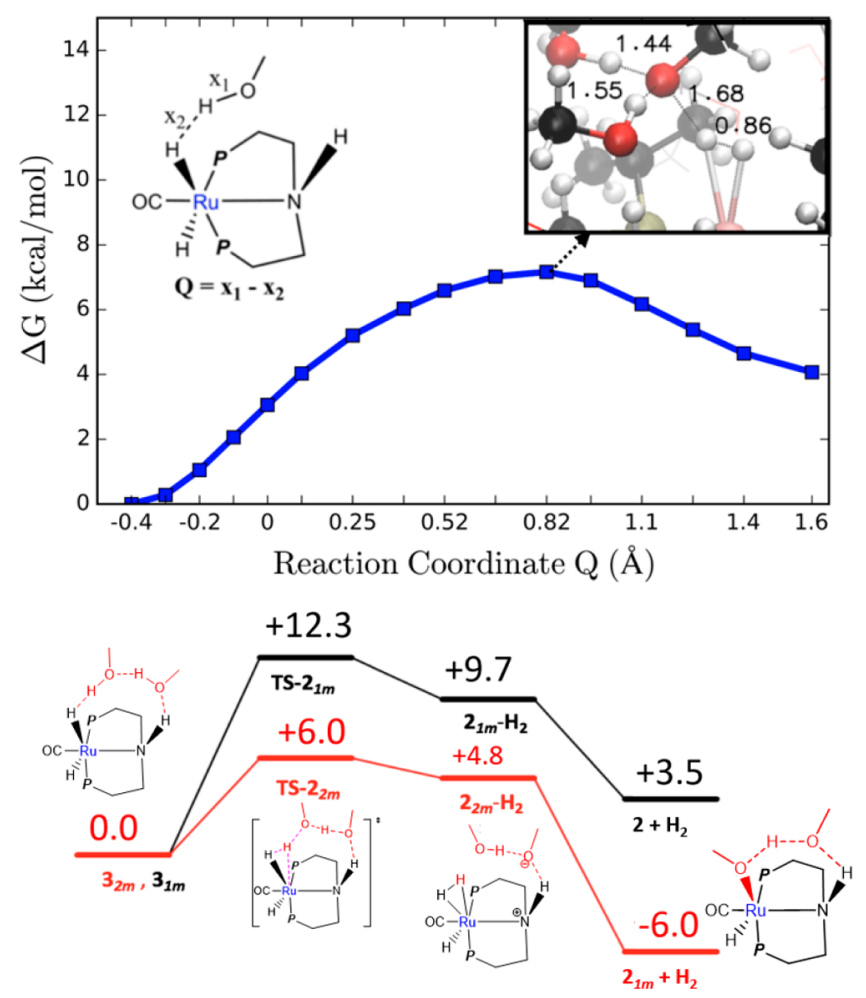

Figure 3. Free energy profile for dihydrogen formation by proton transfer from a solvent $\mathrm{MeOH}$ to the ruthenium hydride, obtained from DFT-MD simulation. The reaction coordinate $Q$ is specified in the left inset. The right inset is a representative configuration near the transition state $(Q=0.82 \AA)$, showing the methoxide anion being stabilized by hydrogen bonds (top) and (bottom) The calculated MERP (Gibbs free energy in $\mathrm{kcal} \mathrm{mol}^{-1}$ ) obtained from static DFT (BP86/def2-TZVP) using an explicit microsolvation model involving one (black) and two (red) additional $\mathrm{MeOH}$ molecules.

transfer. Similarly, it directs the proton of the reacting $\mathrm{MeOH}$ molecule to the metal hydride moiety in a hydrogen-bonding chain involving the $\mathrm{NH}$ moiety of the PNP ligand in TS-2 $2 m$ for hydrogen production. So, if any, it is actually the $\mathrm{NH}$ moiety of the PNP ligand that acts in a cooperative manner with the metal in the key steps of the catalytic cycle rather than the commonly assumed amido moiety. ${ }^{24,25}$ In these key steps, the $\mathrm{NH}$ moiety does not play a direct role.

Evidence for a similar nondirect role of a amido moiety has been reported for metal catalyzed transfer hydrogenation of ketones. ${ }^{22,23,26}$ These observations can be indicative for the more general notion that (de)hydrogenation reactions involving cooperative amido ligands could proceed via reaction steps similar to those observed in the present study when performed in a polar, protic solvent. The $\mathrm{p} K_{\mathrm{a}}$ of the $\mathrm{NH}$ moiety, quantifying its protonation state, will be one of the determining factors for the way it participates in the catalytic cycle.

In summary, we have studied methanol dehydrogenation by a highly active ruthenium pincer complex $\mathbf{1}^{\prime}$ using a combination of $\mathrm{ab}$ initio molecular dynamics and static DFT calculations in the presence of an explicit solvent. The ligand nitrogen of amido complex 1, which was previously believed to be a key catalytic intermediate, was found to have a high proton affinity in polar, protic solvents, resulting in complex $2_{2 m}$ being the actual resting state species of the catalytic cycle. This suggests that the earlier proposed catalytic pathways for 
Scheme 3. Catalytic Pathway with an Explicit Solvation Model for Methanol Oxidation (Top) and (Bottom) Hydrogen Production ${ }^{a}$
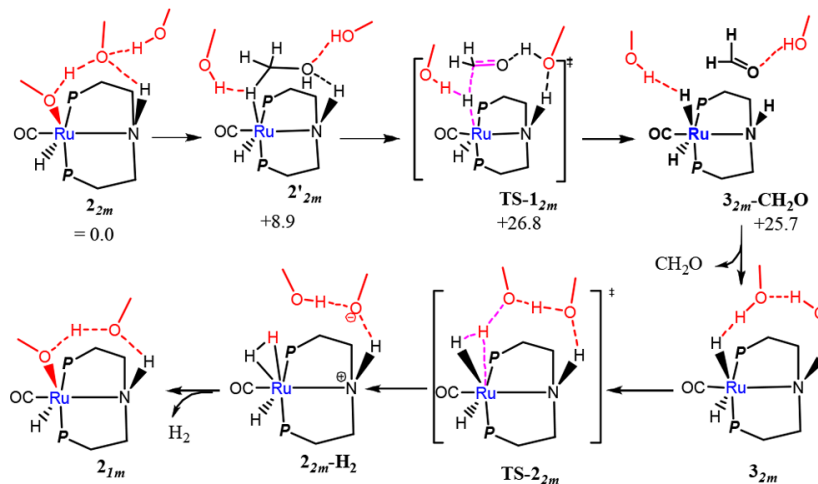

$+8.9$

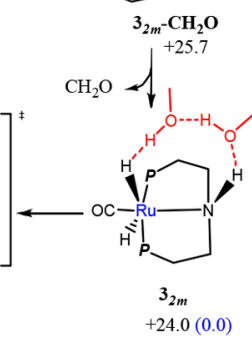

$+18.0(-6.0)$
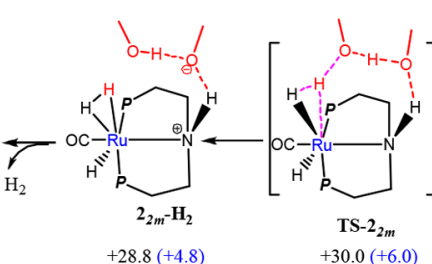

$+28.8(+4.8)$

$$
+30.0(+6.0)
$$

$+24.0(0.0)$

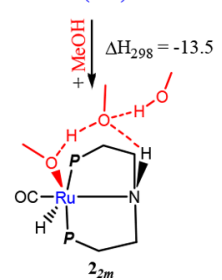

${ }^{a}$ Gibbs free energy values are shown in $\mathrm{kcal} \mathrm{mol}^{-1}$. Note that in the experimental system these reactions are driven by removal of $\mathrm{H}_{2}$ (and $\left.\mathrm{CH}_{2} \mathrm{O}\right)$.

methanol dehydrogenation involving Noyori-type cooperative involvement of a ligand amido moiety are not feasible, as they are based on the existence of deprotonated complexes $2^{-}$and $3^{-}$as resting states. ${ }^{16}$ The stability of these complexes was most likely overestimated in ref 16 . by neglecting the explicit effects of solvent. Our result for the stability of complex $\mathbf{2}_{2 \mathrm{~m}}$ also suggests that the enhancing effect of strong base on the activity of the catalyst is not correlated to the deprotonation of the NH moiety in the PNP ligand. For both the methanol oxidation and the dihydrogen formation steps, DFT-MD simulations show that incorporating a polar, protic solvent substantially influences the relative reaction energies. These differences can be rationalized by considering hydrogen bonding with solvent molecules, which affects the relative energies of the reactants, transition states and products. Static DFT calculations including a small number of explicit solvent molecules can be used to accurately model these same solvent effects, even in a quantitative manner. Our calculations, which account for explicit solvent effects, clearly demonstrate the rate limiting step for methanol dehydrogenation to formaldehyde to be a direct hydride transfer process from the $\mathrm{C}-\mathrm{H}$ bond of a methoxide anion to ruthenium. This observation is in excellent agreement with the need to use high base concentrations in the experimental reactions, as a higher methoxide concentration will of course be beneficial for efficient hydride transfer, lowering the barrier of the rate-limiting step of the catalytic cycle under such nonstandard thermodynamic conditions.

Additionally, the hydrogen production step proceeds via direct protonation of ruthenium hydride moiety by a solvent molecule, while the ligand nitrogen remains protonated throughout the reaction, and therefore cannot act as an internal base for the deprotonation of methanol. Apparently, the "non-innocent/cooperative" amido moiety of the PNP ligand does not seem to play a direct role in catalysis, rather it could facilitate substrate/solvent orientations via hydrogen bonding. This may well be a manifestation of a more general (design) principle for metal-based (de)hydrogenation catalysis in protic solvents.

\section{ASSOCIATED CONTENT}

Supporting Information

The Supporting Information is available free of charge on the ACS Publications website at DOI: 10.1021/acscatal.8b01177.

Details on the molecular dynamics calculations, static DFT calculations, and the $x y z$ coordinates (PDF) Animations of the transition states (ZIP)

\section{- AUTHOR INFORMATION}

\section{Corresponding Authors}

*E-mail: N.Govindarajan@uva.nl.

*E-mail: B.deBruin@uva.nl.

ORCID

Nitish Govindarajan: 0000-0003-3227-5183

Bas de Bruin: 0000-0002-3482-7669

Evert Jan Meijer: 0000-0002-1093-9009

\section{Author Contributions}

${ }^{\S}$ V.S. and N.G. contributed equally to this work. V.S. and N.G. conceived the project. All authors contributed to the writing of the manuscript.

\section{Notes}

The authors declare no competing financial interest.

\section{ACKNOWLEDGMENTS}

This work is part of the Industrial Partnership Programme (IPP) Computational Sciences for Energy Research (projects 13CSER003 and 14CSER044), which is financially supported by The Netherlands Organization for Scientific Research (NWO) and Shell Global International Solutions B.V. V.S and B.d.B acknowledge support from the Research Priority Area Sustainable chemistry of the University of Amsterdam. The calculations were carried out on the Dutch national einfrastructure with the support of the SURF cooperative. We thank Prof. Matthias Beller and Dr. Haijun Jiao for their insightful comments.

\section{REFERENCES}

(1) Nielsen, M.; Alberico, E.; Baumann, W.; Drexler, H.-J.; Junge, H.; Gladiali, S.; Beller, M. Low-Temperature Aqueous-Phase Methanol Dehydrogenation to Hydrogen and Carbon Dioxide. Nature 2013, 495, 85-89.

(2) Rodríguez-Lugo, R. E.; Trincado, M.; Vogt, M.; Tewes, F.; Santiso-Quinones, G.; Grützmacher, H. A Homogeneous Transition Metal Complex for Clean Hydrogen Production from Methanolwater Mixtures. Nat. Chem. 2013, 5, 342-347.

(3) Nielsen, M.; et al. Efficient Hydrogen Production from Alcohols under Mild Reaction Conditions. Angew. Chem., Int. Ed. 2011, 50, 9593-9597.

(4) Fujita, K.; Kawahara, R.; Aikawa, T.; Yamaguchi, R. Hydrogen Production from a Methanol-Water Solution Catalyzed by an Anionic Iridium Complex Bearing a Functional Bipyridonate Ligand under Weakly Basic Conditions. Angew. Chem., Int. Ed. 2015, 54, 9057-9060.

(5) Chakraborty, S.; Lagaditis, P. O.; Förster, M.; Bielinski, E. A.; Hazari, N.; Holthausen, M. C.; Jones, W. D.; Schneider, S. WellDefined Iron Catalysts for the Acceptorless Reversible Dehydrogenation-Hydrogenation of Alcohols and Ketones. ACS Catal. 2014, 4, 3994-4003. 
(6) Hu, P.; Diskin-Posner, Y.; Ben-David, Y.; Milstein, D. Reusable Homogeneous Catalytic System for Hydrogen Production from Methanol and Water. ACS Catal. 2014, 4, 2649-2652.

(7) Campos, J.; Sharninghausen, L. S.; Manas, M. G.; Crabtree, R. $\mathrm{H}$. Methanol Dehydrogenation by Iridium N-Heterocyclic Carbene Complexes. Inorg. Chem. 2015, 54, 5079-5084.

(8) Tanaka, R.; Yamashita, M.; Nozaki, K. Catalytic Hydrogenation of Carbon Dioxide Using Ir(III)-Pincer Complexes. J. Am. Chem. Soc. 2009, 131, 14168-14169.

(9) Wesselbaum, S.; vom Stein, T.; Klankermayer, J.; Leitner, W. Hydrogenation of Carbon Dioxide to Methanol by Using a Homogeneous Ruthenium-phosphine Catalyst. Angew. Chem., Int. Ed. 2012, 51, 7499-7502.

(10) Rohmann, K.; Kothe, J.; Haenel, M. W.; Englert, U.; Hölscher, M.; Leitner, W. Hydrogenation of CO2 to Formic Acid with a Highly Active Ruthenium Acriphos Complex in DMSO and DMSO/Water. Angew. Chem., Int. Ed. 2016, 55, 8966-8969.

(11) Elangovan, S.; Garbe, M.; Jiao, H.; Spannenberg, A.; Junge, K.; Beller, M. Hydrogenation of Esters to Alcohols Catalyzed by Defined Manganese Pincer Complexes. Angew. Chem., Int. Ed. 2016, 55, 15364-15368.

(12) Prichatz, C.; Alberico, E.; Baumann, W.; Junge, H.; Beller, M. Iridium-PNP Pincer Complexes for Methanol Dehydrogenation at Low Base Concentration. Chem CatChem 2017, 9, 1891-1896.

(13) Andérez-Fernández, M.; Vogt, L. K.; Fischer, S.; Zhou, W.; Jiao, H.; Garbe, M.; Elangovan, S.; Junge, K.; Junge, H.; Ludwig, R.; Beller, M. A Stable Manganese Pincer Catalyst for the Selective Dehydrogenation of Methanol. Angew. Chem., Int. Ed. 2017, 56, 559-562.

(14) Annen, S. P.; et al. A Biologically Inspired Organometallic Fuel Cell (OMFC) That Converts Renewable Alcohols into Energy and Chemicals. Angew. Chem., Int. Ed. 2010, 49, 7229-7233.

(15) Yamakawa, M.; Ito, H.; Noyori, R. The Metal-Ligand Bifunctional Catalysis: A Theoretical Study on the Ruthenium(II)Catalyzed Hydrogen Transfer between Alcohols and Carbonyl Compounds. J. Am. Chem. Soc. 2000, 122, 1466-1478.

(16) Alberico, E.; Lennox, A. J. J.; Vogt, L. K.; Jiao, H.; Baumann, W.; Drexler, H.-J.; Nielsen, M.; Spannenberg, A.; Checinski, M. P.; Junge, H.; Beller, M. Unravelling the Mechanism of Basic Aqueous Methanol Dehydrogenation Catalyzed by $\mathrm{Ru}-\mathrm{PNP}$ Pincer Complexes. J. Am. Chem. Soc. 2016, 138, 14890-14904.

(17) Yang, X. Mechanistic Insights into Ruthenium-Catalyzed Production of $\mathrm{H} 2$ and $\mathrm{CO} 2$ from Methanol and Water: A DFT Study. ACS Catal. 2014, 4, 1129-1133.

(18) Vidossich, P.; Lledós, A.; Ujaque, G. First-Principles Molecular Dynamics Studies of Organometallic Complexes and Homogeneous Catalytic Processes. Acc. Chem. Res. 2016, 49, 1271-1278.

(19) Ma, C.; Piccinin, S.; Fabris, S. Reaction Mechanisms of Water Splitting and $\mathrm{H} 2$ Evolution by a $\mathrm{Ru}(\mathrm{II})$-Pincer Complex Identified with Ab Initio Metadynamics Simulations. ACS Catal. 2012, 2, 15001506.

(20) Hodel, F. H.; Luber, S. Redox-Inert Cations Enhancing Water Oxidation Activity: The Crucial Role of Flexibility. ACS Catal. 2016, 6, 6750-6761.

(21) Mattioli, G.; Giannozzi, P.; Amore Bonapasta, A.; Guidoni, L. Reaction Pathways for Oxygen Evolution Promoted by Cobalt Catalyst. J. Am. Chem. Soc. 2013, 135, 15353-15363.

(22) Pavlova, A.; Meijer, E. J. Understanding the Role of Water in Aqueous Ruthenium-Catalyzed Transfer Hydrogenation of Ketones. ChemPhysChem 2012, 13, 3492-3496.

(23) Handgraaf, J.-W.; Meijer, E. J. Realistic Modeling of Ruthenium-Catalyzed Transfer Hydrogenation. J. Am. Chem. Soc. 2007, 129, 3099-3103.

(24) Dub, P. A.; Gordon, J. C. Metal-Ligand Bifunctional Catalysis: The "Accepted" Mechanism, the Issue of Concertedness, and the Function of the Ligand in Catalytic Cycles Involving Hydrogen Atoms. ACS Catal. 2017, 7, 6635-6655.

(25) Dub, P. A.; Henson, N. J.; Martin, R. L.; Gordon, J. C. Unravelling the Mechanism of the Asymmetric Hydrogenation of
Acetophenone by [RuX2(diphosphine)(1,2-Diamine)] Catalysts. J. Am. Chem. Soc. 2014, 136, 3505-3521.

(26) Dub, P. A.; Gordon, J. C. The Mechanism of Enantioselective Ketone Reduction with Noyori and Noyori-Ikariya Bifunctional Catalysts. Dalt. Trans. 2016, 45, 6756-6781. 